\title{
Perfil perinatal asociado a los estados hipertensivos del embarazo
}

\author{
María Inmaculada Sánchez López ${ }^{1}$, Pilar Llabata Carabal2 ${ }^{2}$ Enrique Garrigós Almerich ${ }^{3}$ \\ ${ }^{1}$ Universidad Cardenal Herrera-CEU,CEU Universities. Facultad de Ciencias de la Salud. Valencia. España \\ ${ }^{2}$ Centro de Salud Nápoles y Sicilia. Departamento de Salud de Valencia. Hospital General. Valencia. España \\ ${ }^{3}$ Hospital Francisco de Borja. Servicio de Nefrología. Departamento de Salud de Gandía. Valencia. España
}

\section{Resumen}

Introducción: Los estados hipertensivos del embarazo son un conjunto de trastornos que acontecen en gestantes, siendo una de las principales causas de morbimortalidad materna y perinatal. Se clasifican en: hipertensión crónica, hipertensión gestacional, preeclampsia e hipertensión crónica con preclampsia sobreañadida. EI adecuado control de la presión arterial es clave para su seguimiento.

Objetivos: describir el perfil de los recién nacidos fruto de una gestación con estados hipertensivos y estudiar si existe relación con el tipo de alteración hipertensiva de la madre.

Pacientes y Método: estudio de cohortes retrospectivo, que incluye a recién nacidos de gestantes con estados hipertensivos, seguidas en la unidad de hipertensión arterial, y que naciesen entre el 1 de enero de 2009 y el 31 de diciembre de 2015.

Resultados: la muestra estuvo formada por 84 sujetos. La edad gestacional fue de 37,1 y el peso al nacer fue de $2.782,7 \mathrm{~g}$. Las menores edades gestacionales y peso al nacer fueron en hijos de gestantes con preeclampsia o hipertensión crónica con preeclampsia sobreañadida $(p<0,05)$. El Apgar y el sexo del RN no fue diferente en función del estado hipertensivo materno.

$$
\begin{gathered}
\text { Correspondencia: } \\
\text { María Inmaculada Sánchez López } \\
\text { C/ Pou s/n. 46115 Alfara del Patriarca. Valencia } \\
\text { E-mail: ines_kto@hotmail.com }
\end{gathered}
$$

Conclusiones: el perfil es el de un recién nacido con edad gestacional de 37,1 semanas, peso medio de $2.782,7 \mathrm{~g}$, y Apgar al minuto de nacer de 8,6, y a los 5 minutos de 9,7 . El peso al nacer y la edad gestacional se relacionaron significativamente con el tipo de estado hipertensivo de la madre, describiéndose peores resultados en hijos de gestantes con preeclampsia sola o sobreañadida. número de sesiones de entrenamiento (10 sesiones vs 8,7 sesiones; $p=0,048$ ).

PALABRAS CLAVE: recién nacido; perfil de salud; hipertensión inducida en el embarazo; preeclampsia; epidemiología.

\section{Perinatal profile associated with hypertensive disorders of pregnancy}

\section{Abstract}

Introduction: Pregnant women can be affected with a group of disorders called hypertensive disorders of pregnancy. These are classified as follows: chronic hypertension, gestational hypertension, mild preeclampsia, severe preeclampsia and chronic hypertension with superimposed preeclampsia. Hypertensive disorders of pregnancy are among the leading causes of maternal and perinatal morbidity and mortality. An adequate blood pressure monitoring is essential for the follow-up of this risk group.

Objective: To describe a profile for new-borns delivered in pregnancies with hypertensive disorders of pregnan$c y$, and to determine whether the profile is related to the different types of disorders. 
Patients and Method: Retrospective cohort study, which includes new-borns of pregnant women with hypertensive states, followed in the hypertension unit, and born between January 1, 2009 and December 31, 2015.

Results: The sample consisted of 84 subjects. The gestational age was 37.1 and the birth weight was 2,782.7 g. The lowest gestational ages and birth weight were in children of pregnant women with preeclampsia or chronic hypertension with superimposed preeclampsia $(p<0.05)$. The Apgar test and the sex of the RN was not different based on the maternal hypertensive state.

Conclusions: The profile was a newborn with a gestational age of 37.1 weeks, average weight of $2,782.7 \mathrm{~g}$, and an Apgar test at birth of 8.6, and at 5 minutes of 9.7. Birth weight and gestational age were significantly related to the type of hypertensive state of the mother, with worse results being reported in children of pregnant women with preeclampsia alone or in addition.

KEYWORDS: new-born; health profile; hypertension; pregnancy-induced; pre-eclampsia; epidemiology.

\section{Introducción}

Los estados hipertensivos del embarazo (EHE) son un grupo de trastornos que acontecen en gestantes y afectan a un $5-15 \%$ de todos los embarazos ${ }^{1}$, constituyendo una de las principales causas de morbimortalidad materna y perinatal ${ }^{2}$. Los EHE se clasifican en: hipertensión arterial crónica (HTC), hipertensión gestacional (HTG), preeclampsia (PE) e hipertensión arterial crónica con preeclampsia sobreañadida (HT$\mathrm{C}+\mathrm{PE})^{2,3}$.

La etiología de la PE se asocia a una implantación anómala del embrión en el endometrio, que impide la remodelación fisiológica de las arterias espirales uterinas, permaneciendo éstas con pequeño calibre y alta resistencia vascular, lo que supone un déficit del flujo útero-placentario․․ Esta insuficiencia placentaria provoca una falta de aporte nutricional y respiratorio al feto, que es el causante de un deterioro del crecimiento y bienestar fetal ${ }^{2}$. El parto es el único tratamiento definitivo para la $\mathrm{PE}^{1,2}$. En una gestación sin complicaciones, se recomienda la inducción del mismo cuando el embarazo llega a término²; y ante la aparición de complicaciones se realizará la maduración pulmonar fetal si fuese preciso y se finalizará la gestación intentando que sea después de la semana gestacional $30^{2}$.

En nuestro país, el seguimiento y control de este grupo de riesgo se lleva a cabo por los obstetras en colaboración con el equipo especialista de nefrología. De hecho en muchas de estas gestantes aparece proteinuria y en los casos más graves puede darse fracaso renal agudo ${ }^{4}$. En ese sentido, es adecuado que los profesionales de la salud del área nefrológica conozcan las características de estas alteraciones, de cara al buen cuidado de las gestantes y sus futuros hijos.

Son múltiples los estudios que han descrito el perfil de los recién nacidos ( $R N$ ) de madres con $E H E$, algunos de los cuales analizaron además la relación en función del EHE materno. La mayoría de investigaciones lo hicieron para un único EHE o comparando entre dos EHE. Hasta donde alcanza nuestro conocimiento sólo los estudios de Ferrazzani et al. ${ }^{5}$, Sáez Cantero et al. ${ }^{6}$ y Adu-Bonsaffoh et al. ${ }^{7}$ incluyeron en su muestra todos los EHE, aunque sólo compararon el peso medio del RN y la edad gestacional. En ese sentido, ésta sería la primera publicación que además compara el Apgar, el sexo del RN y el peso medido de forma cualitativa. Por tanto, este estudio pretende en primer lugar describir el perfil perinatal de los EHE y en segundo lugar analizar si dicho perfil se asocia con el tipo de EHE de la madre.

\section{Pacientes y Método}

Estudio observacional analítico longitudinal retrospectivo, realizado en el Hospital Francisco de Borja (hospital público de referencia del Departamento de Salud de Gandía, Valencia). El estudio se llevó a cabo en la unidad de hipertensión arterial (Servicio de Nefrología), que incluye una consulta de enfermería para el seguimiento y atención de pacientes con hipertensión arterial, entre los que se incluyen las participantes del estudio.

Las participantes fueron todas las mujeres atendidas en la citada unidad de hipertensión arterial, con algún EHE y cuyo parto tuviese lugar entre el 1 de enero de 2009 y el 31 de diciembre de 2015. La muestra fueron todos los hijos de la gestación a estudio, siendo el muestreo de tipo no probabilístico consecutivo. Los datos se obtuvieron a partir de la consulta de la historia clínica electrónica de las madres, desde el mes de diciembre de 2014 al de enero de 2016, ambos incluidos. 
Las variables para el perfil perinatal fueron: sexo, edad gestacional, puntuación del test Apgar al minuto y a los 5 minutos de nacer y peso tomándose como variable cuantitativa y cualitativa categorizándose en muy bajo peso $\left(<1.500\right.$ gramos $\left.(\mathrm{g})^{8}\right)$, bajo peso $\left(<2.500 \mathrm{~g}^{8}\right)$, normopeso (2.500-3.999 g) y macrosomía ( $>4.000$ g). Los EHE se clasificaron en: 1) HTC, hipertensión (presión arterial $\geq 140 / 90 \mathrm{mmHg}$ ) que precede al embarazo; 2) $\mathrm{PE}$, hipertensión con proteinuria (proteínas en orina $\geq 300 \mathrm{mg} / 24 \mathrm{~h}$ ), 0 en la ausencia de proteinuria, hipertensión asociada a trombocitopenia (plaquetas $<100 \times 10^{\%} / \mathrm{L}$ ), alteración de la función hepática (transaminasas elevadas al doble de los valores normales), insuficiencia renal (creatinina sérica $>1,1 \mathrm{mg} /$ $\mathrm{dL}$ ), edema pulmonar o nueva aparición de alteraciones visuales/cerebrales; 3) HTG, hipertensión arterial después de la semana 20 de gestación, en ausencia de proteinuria o síntomas sistémicos; 4) $\mathrm{HTC}+\mathrm{PE}$, hipertenisón arterial que ya estaba presente antes del embarazo, asociada a $\mathrm{PE}^{3}$.

El estudio contó con el dictamen favorable del Comité de Investigación, Docencia y Ética del hospital y siguió sus directrices; debido al carácter retrospectivo del estudio, dicho Comité consideró que no era necesario obtener el consentimiento informado, y por tanto se siguieron sus indicaciones. El estudio no presentó ningún conflicto de interés, ni contó con financiación.

Los datos fueron analizados empleando el programa SPSS. En el análisis descriptivo se realizaron medias, desviaciones estándar, rangos y valores mínimos y máximos. La asociación entre el EHE y edad gestacional, peso y Apgar se estudió mediante el test ANOVA. Y la asociación entre el EHE y sexo y peso categorizado se analizó empleando el test Chi cuadrado. Las premisas de normalidad y homogeneidad de varianzas necesarias para el cálculo de la prueba ANOVA, se dieron sólo para el peso del RN, que presentó un comportamiento normal (significación del test Kolmogorov-Smirnov = $0,569>0,05)$ y una homogeneidad de varianzas (significación del test Levene $=0,759>0,05$ ). Pese a que las variables de Apgar y edad gestacional, no presentaron las premisas de normalidad ni homogeneidad de varianzas, se decidió realizar igualmente la prueba ANOVA debido al carácter robusto de la misma, pero teniendo en cuenta que debido al volumen de la muestra los resultados obtenidos debían tomarse con precaución. Los análisis fueron realizados con un intervalo de confianza del 95\%, considerándose significativo un resultado $<0,05$ para la prueba ANOVA y $<0,025$ para el test Chi cuadrado.

\section{Resultados}

Setenta y nueve gestantes participaron en el estudio y dado que 5 gestaciones fueron gemelares, el total de la muestra de RN fue de 84 sujetos. EI EHE más frecuente fue la PE (40/84:47,6\%), seguido de la HTC $(31 / 84: 36,9 \%)$, la HTC+PE (8/84:9,5\%) y la HTG (5/84:6\%).

Un $49,4 \%$ fueron niños y un $50,6 \%$ niñas La edad gestacional media fue de $37,1 \pm 2,6$ semanas, siendo la menor de 31 y la mayor de 41 semanas, con una mediana de 38 semanas. El peso medio fue de $2.782,7 \pm 453,7$ $\mathrm{g}$, con un rango de $2.990 \mathrm{~g}$, siendo el peso más bajo de $1.170 \mathrm{~g}$ y el más elevado de $4.160 \mathrm{~g}$. El normopeso se dio en 47 RN (47/84:56\%), seguido del bajo peso $(30 / 84: 35,7 \%)$, macrosomía $(3 / 84: 3,6 \%)$ y muy bajo peso (2/84:2,4\%). En 2 casos el dato no constaba. La media de Apgar al minuto de nacer fue de $8,6 \pm 1,7$, con un rango de 8 , siendo el Apgar mínimo de 2 y el máximo de 10 . $Y$ a los 5 minutos la media fue de $9,7 \pm 1,1$ con un rango de 8 , donde el Apgar más bajo fue de 2 y el más elevado de 10.

La Tabla 1 muestra el perfil perinatal de los RN y su relación con el EHE. La edad gestacional fue diferente entre los EHE: las menores edades gestacionales se dieron en la PE y la HTC+PE (Figura 1). Las diferencias significativas pareadas fueron: HTC frente a PE $(38,8$ vs. 36,2 semanas; $p=0,000)$ e HTC frente a HT$C+P E(38,8$ vs. 35 semanas; $p=0,029)$. El peso también fue diferente según el EHE materno, tanto cuando se analizó de forma cuantitativa como categórica. El peso medio fue mayor en la HTC, seguido de HTG, PE e HTC+PE (figura 1). Las diferencias significativas

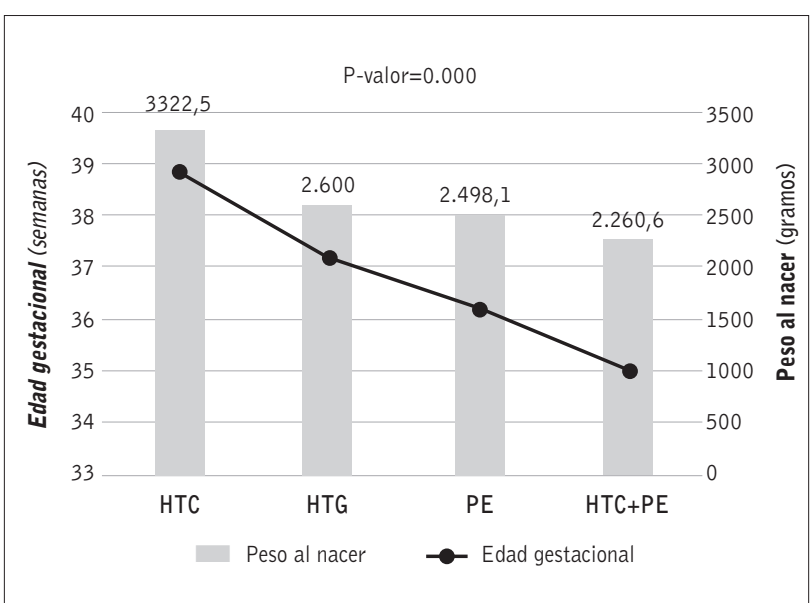

Figura 1: Edad gestacional y peso al nacer en los diferentes estados hipertensivos. 
Tabla 1. Relación entre el perfil perinatal y los diferentes EHE.

\begin{tabular}{|c|c|c|c|c|c|}
\hline \multirow[b]{2}{*}{ Perfil perinatal } & \multicolumn{4}{|c|}{ EHE } & \multirow[b]{2}{*}{ P valor } \\
\hline & HTC & HTG & PE & HTC+PE & \\
\hline \multicolumn{6}{|l|}{ Sexo } \\
\hline Niño & $14(34,1 \%)$ & $5(12,2 \%)$ & $18(43,9 \%)$ & $4(9,8 \%)$ & \multirow[t]{2}{*}{ NS } \\
\hline Niña & $17(40,5 \%)$ & $0(0 \%)$ & $21(50 \%)$ & $4(9,5 \%)$ & \\
\hline $\begin{array}{l}\text { Edad gestacional } \\
\text { (semanas)a }^{a}\end{array}$ & $\begin{array}{c}38,8 \pm 1,3 \\
(38,3-39,2)\end{array}$ & $\begin{array}{l}37,2 \pm 1,5 \\
(35,4-39)\end{array}$ & $\begin{array}{c}36,2 \pm 2,9 \\
(35,3-37,1)\end{array}$ & $\begin{array}{c}35,0 \pm 2,7 \\
(32,8-37,2)\end{array}$ & $0,000^{b}$ \\
\hline $\begin{array}{l}\text { Peso al nacer } \\
\text { (gramos) }^{\mathrm{a}}\end{array}$ & $\begin{array}{c}3.322,5 \pm 536,3 \\
(3.122,3 \quad 3.522,7)\end{array}$ & $\begin{array}{c}2.600 \pm 510,4 \\
(1.966,3 \quad 3.233 .7)\end{array}$ & $\begin{array}{c}2.498,1 \pm 705,2 \\
(2.269,5 \quad 2.726,7)\end{array}$ & $\begin{array}{c}2.260,6 \pm 705,6 \\
(1.670,7 \quad 2.850,5)\end{array}$ & $0,000^{b}$ \\
\hline \multicolumn{6}{|l|}{$\begin{array}{l}\text { Peso al nacer } \\
\text { (categorías) }\end{array}$} \\
\hline Muy bajo peso & $0(0 \%)$ & $0(0 \%)$ & $1(2,6 \%)$ & $1(12,5 \%)$ & \multirow{4}{*}{$0,003^{c}$} \\
\hline Bajo peso & $2(6,7 \%)$ & $2(40 \%)$ & $22(56,4 \%)$ & $4(50 \%)$ & \\
\hline Normopeso & $26(86,7 \%)$ & $3(60 \%)$ & $15(38,5 \%)$ & $3(37,5 \%)$ & \\
\hline Macrosomía & $2(6,7 \%)$ & $0(0 \%)$ & $1(2,6 \%)$ & $0(0 \%)$ & \\
\hline Apgar a 1 minuto ${ }^{a}$ & $9,0 \pm 1,4(8,5-9,5)$ & $7,4 \pm 1,7(5,32-9,5)$ & $8,5 \pm 1,9(7,8-9,2)$ & $8,5 \pm 0,8(7,6-9,4)$ & NS \\
\hline Apgar 5 minutos $^{\mathrm{a}}$ & $9,7 \pm 1,4(9,2-10,3)$ & $9,0 \pm 1,0(7,8-10,2)$ & $9,7 \pm 0,8(9,4-10)$ & $9,8 \pm 0,4(9,4-10,3)$ & NS \\
\hline
\end{tabular}

EHE: estados hipertensivos del embarazo; HTC: hipertensión crónica; HTG: hipertensión gestacional; PER: preeclampsia.

a: media desviación estándar ( $95 \%$ intervalo de confianza).

b: ANOVA significación si p<0,05.

c: Chi cuadrado significación si $p<0,025$.

pareadas fueron: HTC frente a PE $(3.322,5 \mathrm{~g}$ vs. 2.498 $g ; p=0,000)$ e HTC frente a HTC+PE $(3.322,5 \mathrm{~g}$ frente a $2.260,6 \mathrm{~g} ; \mathrm{p}=0,018)$. Al categorizar el peso del RN se observó que los EHE con mayor porcentaje de bajo peso fueron la HTC+PE (50\%) y la PE $(56,4 \%)$, describiéndose casos de muy bajo peso sólo en estos subgrupos.

El Apgar y el sexo no fueron diferentes en función del EHE materno.

\section{Discusión}

Este estudio retrospectivo ha descrito diferencias significativas en el peso y la edad gestacional en función del EHE materno. Además, el perfil del RN para estas dos variables se encuentra próximo a los límites de anormalidad; por tanto, el seguimiento y los cuidados enfermeros deben adecuarse a esta población de riesgo.

Como indican Fernández et al. ${ }^{9}$, la consulta de enfermería en hipertensión arterial puede ayudar al buen control de la enfermedad hipertensiva y de las complicaciones asociadas a la misma, ayudando por otra parte a reforzar el cumplimiento. En esa línea, la con- sulta de enfermería de la unidad de hipertensión arterial que en nuestro centro atiende a las gestantes con $E H E$, contribuye a una mejora en el seguimiento y atención de este colectivo de riesgo, sobre todo en lo referente a 2 áreas: el control de los valores de presión arterial (función interdependiente) y el cuidado mediante la aplicación de intervenciones enfermeras (función independiente).

En la consulta de enfermería, una de las actividades que se lleva a cabo es la medición de la presión arterial (3 series de medidas) y la comparación de los valores obtenidos con el registro de automonitorización de presión arterial (AMPA) que la gestante ha realizado en su domicilio. En caso de que se detecten cifras dispares (posible reacción de alerta o fenómeno de "bata blanca"), la enfermera valorará si es adecuado realizar una monitorización ambulatoria de la presión arterial (MAPA). La correcta medición de la presión arterial es fundamental en este grupo de riesgo ya que las cifras obtenidas serán empleadas por el nefrólogo para diagnosticar el tipo de $\mathrm{EHE}^{1,2}$ y servirán de base para la instauración o modificación del tratamiento farmacológico hipotensor ${ }^{1,2,4}$. En relación a este último, su objetivo es evitar complicaciones vasculares asociadas a un 
aumento de la presión arterial, pero teniendo en cuenta no disminuirla tanto que afecte al riego útero-placentario; en ese sentido, se aconseja que en la PE la presión arterial sistólica se encuentre entre $140-160 \mathrm{mmHg}$ y la diastólica entre 90 y $100 \mathrm{mmHg}$ evitando que baje de $140 / 90 \mathrm{~mm} \mathrm{Hg}^{2}$. Por otra parte, las cifras de presión arterial también son tenidas en cuenta de cara a decidir sobre la finalización del embarazo ${ }^{1,4}$.

En segundo lugar, la consulta de enfermería puede instituirse como el lugar idóneo para reforzar el cumplimiento terapéutico y para la educación ${ }^{9}$. En concreto, en las gestantes con EHE se observan dos intervenciones enfermeras fundamentales: en primer lugar, los cuidados del embarazo de alto riesgo (NIC 6800), que incluyen entre sus actividades principales: fomentar que la gestante exprese sus sentimientos o miedos asociados al bienestar fetal o la seguridad personal, instruir sobre el uso de los fármacos prescritos, instruir sobre las técnicas de la AMPA, facilitarle guía sobre los signos y síntomas que requieren atención médica urgente (elevación de las cifras de presión arterial, dolor epigástrico, cefalea severa, alteraciones visuales, edemas o hemorragia vaginal). Y en segundo lugar, la enseñanza del proceso de enfermedad (NIC 5602), entre cuyas actividades se incluyen: evaluar el nivel de conocimientos sobre su enfermedad y explicarla en caso necesario, describirle el fundamento de las recomendaciones $y / 0$ la terapia que le hayan prescrito e instruir a la gestante sobre los signos y síntomas de alarma sobre los que debe informar ${ }^{10}$.

El seguimiento a este colectivo de riesgo, debe hacerse más riguroso si cabe en aquellos EHE más graves ( $P E$ o HTC+PE), ya que se asocian a peores resultados perinatales como se ha descrito, y del mismo modo que reportan investigaciones anteriores, como se describe a continuación. Respecto a la edad gestacional en el momento del parto, un recién nacido prematuro es aquel que nace de forma pretérmina antes de la semana 37 de embarazo ${ }^{8}$. La edad gestacional de nuestra muestra $(37,1$ semanas $)$ se aproxima mucho al límite de embarazo pretérmio. Esto podría explicarse porque los EHE son una de las primeras causas de prematuridad ${ }^{1,2,8}$; de hecho, la edad gestacional media de los subgrupos de PE e HTC+$\mathrm{PE}$ corresponde a RN prematuros. Ferrazanni et al.5 también describen las menores edades gestacionales en la $\mathrm{HTC}+\mathrm{PE}$ y la $\mathrm{PE}$, seguidas de la HTC e HTG $(33,9 \pm 3,8,35,4 \pm 3,5,37,8 \pm 2,9$ y $38,2 \pm 2,7$ semanas respectivamente). Para aquellos estudios que comparan entre 2 ó 3 EHE, los resultados son similares a los nuestros: menor edad gestacional en PE que en HTG (37 semanas vs. $38 ; p<0,00^{11}$ y $37,6 \pm 2,9$ semanas vs. $\left.39,6 \pm 0,9 ; p<0,00^{12}\right)$; y en PE que en HTC o HTG $\left(37,8,38\right.$ y 39,1 semanas respectivamente $\left.{ }^{13}\right)$. Por el contrario, Cruz et al..$^{14}$ describen medias similares entre estos subgrupos $(37,2 \pm 3,2,37,2 \pm 3,2$ y $37,4 \pm 2,8$ semanas respectivamente).

Por otra parte, el peso medio descrito para el total de RN $(2.782,7 \pm 453,7 \mathrm{~g})$ se aproxima a $2.500 \mathrm{~g}$, que es el límite considerado para RN de bajo peso, y que se relaciona entre otros motivos con la hipertensión en gestantes $^{1}$; nuestros resultados muestran que el peso medio en la PE e HTC+PE es menor de $2.500 \mathrm{~g}$, y que estos dos subgrupos presentan las mayores tasas de RN con muy bajo peso o bajo peso. Del mismo modo, Ferrazzani et al..$^{5}$ y Sáez Cantero et al. ${ }^{6}$ describen los pesos al nacer más bajos en estos subgrupos y Adu-Bonsaffoh et al. ${ }^{7}$ reportan las mayores tasas de bajo peso en ellos en comparación con la HTG e HTC. Cuando el peso se compara sólo para algunos $E H E$, los resultados también son similares: menor peso en PE que en $\mathrm{HTG}^{11,12}$ y menor en PE que en $\mathrm{HTC}_{\mathrm{T}} \mathrm{HTG}^{13}$. En cambio otras investigaciones muestran valores similares en la $\mathrm{HTC}, \mathrm{HTG}$ y $\mathrm{PE}^{14}$.

La media de Apgar a los 5 minutos es mayor que al minuto de nacer, tanto para el total de RN, como para cada uno de los subgrupos. Madi et al. ${ }^{15}$ también describen este resultado en su estudio realizado a gestantes con HTC, que presentan medias de Apgar de 7,3 $\pm 2,2$ al minuto y de $8,7 \pm 1,5$ a los 5 minutos.

Los datos incompletos de las historias clínicas, son una limitación típica de los estudios retrospectivos que debe tenerse en cuenta; no obstante, sólo hubo 2 casos incompletos para el peso al nacer y 8 para el Apgar. Otra limitación se asocia con las premisas de normalidad y homogeneidad de varianzas, que no se dieron en el Apgar y la edad gestacional; aunque el test ANOVA es robusto estadísticamente, los resultados de estas pruebas deben tomarse con precaución.

A la vista de estos datos podemos concluir que el perfil de la muestra es el de un RN con edad gestacional de 37,1 semanas, sin diferencias de sexo, con peso medio de $2.782,7 \mathrm{~g}$, Apgar al minuto de nacer de 8,6, y a los 5 minutos de 9,7 .

Los hijos de gestantes con PE o HTC+PE presentaron el peor perfil perinatal para la edad gestacional y el peso, comparados con los otros EHE, con diferencias significativas. 
Se recomienda un seguimiento de estas gestantes de riesgo en la consulta de enfermería de hipertensión arterial.

\section{Agradecimientos}

Agradecemos al Comité de Investigación, Docencia y Ética del Hospital Francisco de Borja la aprobación de este trabajo para ser realizado.

\section{Los autores declaran que no hay conflicto de interés.}

Recibido: 26 abril 2018

Revisado: 5 mayo 2018

Modificado: 20 mayo 2018

Aceptado: 30 junio 2018

\section{Bibliografía}

1. Jim B, Sharma S, Kebede T et al. Hypertension in pregnancy: a comprehensive update. Cardiol Rev [Internet] 2010 Julio-Agosto [Consultado 24 enero 2018];18(4):178-89. Disponible en: http://www. ncbi.nlm.nih.gov/pubmed/20539101.

2. Cararach V, Bartha J, Gratacós E, Bellart J, Iglesias Diz $\mathrm{M}$, Comino $\mathrm{R}$ et al. Estados hipertensivos del embarazo. $1^{a}$ ed. España: Nabe editores; 2008. p. 143-05.

3. Roberts JM, August PA, Bakris G, Barton JR, Bernstein IM, Druzin M et al. (Task Force on Hypertension in Pregnancy: American College of Obstetricians and Gynecologists'). Hypertension in pregnancy. Obstetrics \& Gynecology [Internet] 2013 Noviembre [Consultado 24 enero 2018]; 122:1122-1131. Disponible en: 10.1097/01. AOG.0000437382.03963.88.

4. Marín Iranzo R, Gorostidi Pérez M, Álvarez Navascués R. Hipertensión arterial y embarazo. NefroPlus [Internet] 2011 Junio [Consultado 25 abril 2018];4(2):21-30. Disponible en: http://

www.revistanefrologia.com/es-publicacion-nefroplus-articulo-hipertension-arterial-embarazo-X1888970011001019.

5. Ferrazzani S, Luciano R, Garofalo S, D'Andrea V, De Carolis S, De Carolis M et al. Neonatal outcome in hypertensive disorders of pregnancy. Early Hum Dev [Internet] 2011 Marzo [Consultado 12 febrero 2018];87(6):445-9. Disponible en: https://www.sciencedirect.com/science/article/pii/ S0378378211001460?via\%3Dihub.

6. Sáez Cantero V, Pérez Hernández MT, Agüero Alfonso G, González García H, Alfonso Dávila A. Resultados perinatales relacionados con trastornos hipertensivos del embarazo. Rev Cubana Obstet Ginecol [Internet] 2012 Enero-Marzo [Consultado 24 enero 2018]; 38(1):36-44. Disponible en: http://scielo.sld.cu/scielo.php?script=sci_arttext\&pid=S0138-600X2012000100005.

7. Adu-Bonsaffoh K, Ntumy MY, Obed SA, Seffah JD. Perinatal outcomes of hypertensive disorders in pregnancy at a tertiary hospital in Ghana. BMC Pregnancy Childbirth [Internet] 2017 Noviembre [Consultado 12 febrero 2018]; 17(1): [aprox. 7 p.] Disponible en: https://www.ncbi.nlm.nih.gov/ pmc/articles/PMC5696910/pdf/12884_2017_Article_1575.pdf.

8. Rellan Rodríguez S, García de Ribera C, Aragón Garcia M. El recién nacido prematuro. España: Asociación Española de Pediatría; 2008. p. 6975. [Consultado 29 agosto 2018]. Disponible en: https://www.aeped.es/sites/default/files/documentos/8_1.pdf.

9. Fernández L, Guerrero L, Segura J, Gorostidi M. Papel del personal de enfermería en el control de la hipertensión arterial y en la investigación cardiovascular. Hipertens Riesgo Vasc [Internet] 2010 Enero [Consultado 29 agosto 2018]; 27 (Supl 1): 41-52. Disponible en: http://www.elsevier.es/es-revista-hipertension-riesgo-vascular-67-pdf-S188918 3710700088-S300.

10. Bulechek G, Butcher H, Dochterman J, Wagner C. Clasificación de Intervenciones de Enfermería (NIC). $6^{a}$ ed. España: Elsevier; 2013.

11. Romero Arauz J, Ortiz Díaz C, Leaños Miranda A, Martínez Rodríguez 0. Evolución de hipertensión 
gestacional a preeclampsia. Ginecol Obstet Mex [Internet] 2014 Abril [Consultado 25 abril 2018]; 82(4):229-235. Disponible en: https://ginecologiayobstetricia.org. $\mathrm{mx} / \mathrm{secciones/articulos-origina-}$ les-numero83/evolucion-de-hipertension-gestacional-a-preeclampsia/.

12. Bellomo G, Venanzi S, Saronio P, Verdura C, Narducci PL. Prognostic significance of serum uric acid in women with gestational hypertension. Hypertension [Internet] 2011 Agosto [Consultado 24 enero 2018]; 58(4):704-708. Disponible en: http://hyper. ahajournals.org/content/58/4/704.long.

13. Browne J, Vissers K, Antwi E, Srofenyoh E, Van der Linden $\mathrm{E}$, Agyepong I et al. Perinatal outcomes after hypertensive disorders in pregnancy in a low resource setting. TMIH [Internet] 2015 Diciembre [Consultado 24 enero 2018]; 20(12):17781786. Disponible en: http://onlinelibrary.wiley.com/ doi/10.1111/tmi.12606/full.
14. Cruz MO, Gao W, Hibbard JU. Obstetrical and perinatal outcomes among women with gestational hypertension, mild preeclampsia, and mild chronic hypertension. Am J Obstet Gynecol [Internet] 2011 Junio [Consultado 12 febrero 2018]; 205(3): [aprox. 9 p.] Disponible en: https://www.sciencedirect.com/ science/article/pii/S0002937811007617?via\%3Dihub.

15. Madi M, Araújo B, Zatti $H$, Rombaldi R, de Zorzi P, Terres $A$ et al. Chronic Hypertension and Pregnancy at a Tertiary-Care and University Hospital. Hypertens Pregnancy [Internet] 2012 Diciembre [Consultado 24 enero 2018]; 31(3):350-356. Disponible en: http://www.tandfonline.com/doi/ $\mathrm{abs} / 10.3109 / 10641955.2010 .525279$ ?journalCode=ihip20.

Este artículo se distribuye bajo una Licencia Creative Commons Atribución-NoComercial 4.0 Internacional. https://creativecommons.org/licenses/by-nc/4.0/ 